\title{
Point and contact equivalence groupoids of two-dimensional quasilinear hyperbolic equations
}

\author{
Roman O. Popovych \\ Fakultät für Mathematik, Universität Wien, Oskar-Morgenstern-Platz 1, 1090 Wien, Austria \\ Institute of Mathematics of NAS of Ukraine, 3 Tereshchenkivska Str., 01024 Kyiv, Ukraine \\ E-mail: rop@imath.kiev.ua
}

\begin{abstract}
We describe the point and contact equivalence groupoids of an important class of twodimensional quasilinear hyperbolic equations. In particular, we prove that this class is normalized in the usual sense with respect to point transformations, and its contact equivalence groupoid is generated by the first-order prolongation of its point equivalence groupoid, the contact vertex group of the wave equation and a family of contact admissible transformations between trivially Darboux-integrable equations.
\end{abstract}

\section{Introduction}

Genuine (first-order) contact transformations between differential equations [9, 12], are rarer objects than their point counterparts. These transformations were introduced by Sophus Lie. In particular, it was known to him [3] that the class of Monge-Ampére equations is closed under the action of its contact equivalence (pseudo)group, which consists of all contact transformations of two independent and one dependent variables. In the modern terminology $[10,11,13]$, this means that this class is normalized in the usual sense with respect to contact transformations. The well-known Bäcklund theorem [9, Theorem 4.32] states that genuine contact transformations, which are not the first-order prolongations of point transformations, exist only in the case of one dependent variable.

In the present paper, we compute the point and contact equivalence groupoids of the class $\mathcal{H}_{\text {gen }}$ of two-dimensional quasilinear hyperbolic equations of the form

$$
u_{x y}=f\left(x, y, u, u_{x}, u_{y}\right),
$$

which is properly contained in the class of Monge-Ampére equations. Here $x$ and $y$ are the independent variables, $u$ is the unknown function of $(x, y)$, which is treated as the dependent variable, and the arbitrary element $f$ of the class runs through the set $\mathcal{A}$ of differential functions for these variables that depend at most on $\left(x, y, u, u_{x}, u_{y}\right)$, i.e., whose order is less than or equal to one. Subscripts of functions denote derivatives with respect to the corresponding variables, e.g., $u_{x y}:=\partial^{2} u / \partial x \partial y$ and $f_{u_{x}}:=\partial f / \partial u_{x}$, and we use the same notation for the derivatives $u_{x}, u_{y}, u_{x x}, u_{x y}$ and $u_{y y}$ and for the corresponding coordinates of the second-order jet space $\mathrm{J}^{2}\left(\mathbb{R}_{x, y}^{2}, \mathbb{R}_{u}\right)$ with the independent variables $(x, y)$ and the dependent variable $u$. The consideration is within the local framework. We employ the terms "group" and "groupoid" for pseudogroups and pseudogroupoids, respectively. For a fixed value of the arbitrary element $f$, let $\mathcal{E}_{f}, G_{f}^{\mathrm{c}}$ and $\mathcal{G}_{f}^{\mathrm{c}}$ denote the equation from the class $\mathcal{H}_{\text {gen }}$ with this value of $f$, its contact symmetry group and its contact vertex group, $\mathcal{G}_{f}^{\mathrm{c}}:=\left\{(f, \Phi, f) \mid \Phi \in G_{f}^{\mathrm{c}}\right\}$. See [13] for necessary notions of the theory of admissible transformations within classes of differential equations. The space with coordinates $\left(x, y, u, u_{x}, u_{y}\right)$ is the minimal underlying space for both the point and the contact equivalence groups of the class $\mathcal{H}_{\text {gen }}$, cf. [2, footnote 1] and [10, footnote 1]. By $\mathrm{D}_{x}$ and $\mathrm{D}_{y}$ we denote the operators of total derivatives with respect to $x$ and $y$, respectively, $\mathrm{D}_{x}=\partial_{x}+u_{x} \partial_{u}+u_{x x} \partial_{u_{x}}+u_{x y} \partial_{u_{y}}+\cdots$ and $\mathrm{D}_{y}=\partial_{y}+u_{y} \partial_{u}+u_{x y} \partial_{u_{x}}+u_{y y} \partial_{u_{y}}+\cdots$.

The form (1) is the most general form for equations that can be interpreted as $(1+1)$ dimensional generalized nonlinear Klein-Gordon equations in the light-cone (characteristic) coordinates. It is also the canonical form for single second-order two-dimensional partial differential 
equations each of whose two Monge systems admits a first integral of the first order [12, Theorem 12.1.2]. The class $\mathcal{H}_{\text {gen }}$ contains a number of famous equations, including the wave equation, the Klein-Gordon equation, the Liouville equation, the Tzitzeica equation and the sine-Gordon (or Bonnet) equation. The problem of studying the subclass of equations of the form (1) with $f_{u_{x}}=f_{u_{y}}=0$ within the framework of group analysis of differential equations was posed by Sophus Lie [7]. The group classification problem for this subclass was completely solved in [1]; see also a review of results on this and other subclasses of $\mathcal{H}_{\text {gen }}$ therein. In $[5,6]$, Lie symmetries had been classified for the wider subclass of $\mathcal{H}_{\text {gen }}$ that consists of the equations of the form (1) with $f_{u_{x}}=f_{u_{y}}$, which had been originally presented in the regular space-time variables, and the part of this classification for the above narrower subclass was enhanced in [1]; see the discussion in the conclusions of $[1,13]$. The equations from the entire class $\mathcal{H}_{\text {gen }}$ for which each of the two Monge systems admits at least two functionally independent first integrals are called hyperbolic Goutsat equations. Such equations were classified by Goutsat with respect to the equivalence generated by the point equivalence group $G_{\text {gen }}^{\sim}$ of the class $\mathcal{H}_{\text {gen }}$ (this group is given in Theorem 2 below). See [12, Chapter 13] for the presentation of the enhanced classification proof by Vessiot and [4] for references on modern results related to Darboux-integrable equations.

We describe contact admissible transformations within the class $\mathcal{H}_{\text {gen }}$, which essentially generalizes Lie's study in [7]. After deriving determining equations for such transformations in Section 2, we compute point and contact equivalence groupoids of the class $\mathcal{H}_{\text {gen }}$ in Section 3. In particular, we prove that both the sources and targets of genuine contact admissible transformations are trivially Darboux-integrable equations.

\section{Determining equations for contact admissible transformations}

Applying the direct method of finding admissible transformations, we fix a contact admissible transformation $\mathcal{T}=(f, \Phi, \tilde{f})$ in the class $\mathcal{H}_{\text {gen }}$. Here the equations $\mathcal{E}_{f}: u_{x y}=f\left(x, y, u, u_{x}, u_{y}\right)$ and $\mathcal{E}_{\tilde{f}}: \tilde{u}_{\tilde{x} \tilde{y}}=\tilde{f}\left(\tilde{x}, \tilde{y}, \tilde{u}, \tilde{u}_{\tilde{x}}, \tilde{u}_{\tilde{y}}\right)$ belong to the class $\mathcal{H}_{\text {gen }}$, where $f \in \mathrm{C}^{\infty}(\Omega, \mathbb{R})$ and $\tilde{f} \in \mathrm{C}^{\infty}(\tilde{\Omega}, \mathbb{R})$ with connected open domains $\Omega$ and $\tilde{\Omega}$ contained in $\mathbb{R}^{5}$. $\Phi$ is a contact transformation with the independent variables $(x, y)$ and the dependent variable $u, \Phi:\left(\tilde{x}, \tilde{y}, \tilde{u}, \tilde{u}_{\tilde{t}}, \tilde{u}_{\tilde{y}}\right)=\left(X, Y, U, U^{x}, U^{y}\right)$, that is defined by a diffeomorphism from $\Omega$ onto $\tilde{\Omega}$ and maps $\mathcal{E}_{f}$ to $\mathcal{E}_{\tilde{f}}, \Phi_{*} \mathcal{E}_{f}=\mathcal{E}_{\tilde{f}}$. Thus, the functions $X, Y, U, U^{x}$ and $U^{y}$ in the components of the transformation $\Phi$ are smooth functions of $z:=\left(x, y, u, u_{x}, u_{y}\right) \in \Omega$ with $\mathrm{J}:=\left|\partial\left(X, Y, U, U^{x}, U^{y}\right) / \partial\left(x, y, u, u_{x}, u_{y}\right)\right| \neq 0$ that satisfy the contact condition

$$
\begin{aligned}
& U^{x} \mathrm{D}_{x} X+U^{y} \mathrm{D}_{x} Y=\mathrm{D}_{x} U, \\
& U^{x} \mathrm{D}_{y} X+U^{y} \mathrm{D}_{y} Y=\mathrm{D}_{y} U .
\end{aligned}
$$

Collecting coefficients of the second derivatives of $u$ in this condition leads to the system

$$
\begin{array}{ll}
U^{x} X_{u_{x}}+U^{y} Y_{u_{x}}=U_{u_{x}}, & U^{x} \hat{\mathrm{D}}_{x} X+U^{y} \hat{\mathrm{D}}_{x} Y=\hat{\mathrm{D}}_{x} U, \\
U^{x} X_{u_{y}}+U^{y} Y_{u_{y}}=U_{u_{y}}, & U^{x} \hat{\mathrm{D}}_{y} X+U^{y} \hat{\mathrm{D}}_{y} Y=\hat{\mathrm{D}}_{y} U,
\end{array}
$$

where $\hat{\mathrm{D}}_{x}=\partial_{x}+u_{x} \partial_{u}$ and $\hat{\mathrm{D}}_{y}=\partial_{y}+u_{y} \partial_{u}$ are the truncated operators of total derivatives with respect to $x$ and $y$, respectively.

Lemma 1. Up to the discrete equivalence transformation $\mathcal{J}^{0}: \tilde{x}=y, \tilde{y}=x, \tilde{u}=u, \tilde{u}_{\tilde{x}}=u_{y}$, $\tilde{u}_{\tilde{y}}=u_{x}, \tilde{f}=f$ of the class $\mathcal{H}_{\text {gen }}$, the components of the transformational part $\Phi$ of any contact admissible transformation $\mathcal{T}$ in this class satisfy, on the entire corresponding domain $\Omega$, the joint system of (3) and

$$
\begin{aligned}
& \hat{\mathrm{D}}_{y} X+f X_{u_{x}}=0, \quad X_{u_{y}}=0, \quad U_{u_{y}}^{x}=\left(\Phi^{*} \tilde{f}\right) Y_{u_{y}}, \quad \hat{\mathrm{D}}_{y} U^{x}+f U_{u_{x}}^{x}=\left(\Phi^{*} \tilde{f}\right) \hat{\mathrm{D}}_{y} Y, \\
& \hat{\mathrm{D}}_{x} Y+f Y_{u_{y}}=0, \quad Y_{u_{x}}=0, \quad U_{u_{x}}^{y}=\left(\Phi^{*} \tilde{f}\right) X_{u_{x}}, \quad \hat{\mathrm{D}}_{x} U^{y}+f U_{u_{y}}^{y}=\left(\Phi^{*} \tilde{f}\right) \hat{\mathrm{D}}_{x} X .
\end{aligned}
$$


Proof. We expand the condition $\Phi_{*} \mathcal{E}_{f}=\mathcal{E}_{\tilde{f}}$ via substituting the expression for the involved jet variables with tildes in terms of the jet variables without tildes,

$$
\left|\begin{array}{cc}
\mathrm{D}_{x} U^{y} & \mathrm{D}_{y} U^{y} \\
\mathrm{D}_{x} Y & \mathrm{D}_{y} Y
\end{array}\right|=\left|\begin{array}{cc}
\mathrm{D}_{x} X & \mathrm{D}_{y} X \\
\mathrm{D}_{x} U^{x} & \mathrm{D}_{y} U^{x}
\end{array}\right|=\left(\Phi^{*} \tilde{f}\right)\left|\begin{array}{cc}
\mathrm{D}_{x} X & \mathrm{D}_{y} X \\
\mathrm{D}_{x} Y & \mathrm{D}_{y} Y
\end{array}\right| \quad \text { on solutions of } \mathcal{E}_{f} .
$$

Here $\Phi^{*}$ denotes the pullback by $\Phi, \Phi^{*} \tilde{f}:=\tilde{f}\left(X, Y, U, U^{x}, U^{y}\right)$. The first equality in (5) is a differential consequence of the system (2). Define two $2 \times 4$ smooth matrix functions on $\Omega$,

$$
A_{1}:=\left(\begin{array}{c}
\mathfrak{Q} X \\
\mathfrak{Q} U^{x}-\left(\Phi^{*} \tilde{f}\right) \mathfrak{Q} Y
\end{array}\right)_{\mathfrak{Q} \in \mathcal{M}} \quad \text { and } \quad A_{2}:=\left(\begin{array}{c}
\mathfrak{Q} Y \\
\mathfrak{Q} U^{y}-\left(\Phi^{*} \tilde{f}\right) \mathfrak{Q} X
\end{array}\right)_{\mathfrak{Q} \in \mathcal{M}},
$$

where $\mathcal{M}:=\left(\partial_{u_{x}}, \partial_{u_{y}}, \hat{\mathrm{D}}_{x}+f \partial_{u_{y}}, \hat{\mathrm{D}}_{y}+f \partial_{u_{x}}\right)$.

It is obvious that the set $\Omega_{1}:=\left\{z \in \Omega \mid \operatorname{rank} A_{1}(z)=\operatorname{rank} A_{2}(z)=2\right\}$ is open. Moreover, it is dense in $\Omega$. Indeed, $\Omega_{1}=\Omega \backslash\left(\Omega_{01} \cup \Omega_{02} \cup \Omega_{03} \cup \Omega_{04}\right)$, where

$$
\begin{array}{ll}
\Omega_{01}:=\{z \in \Omega \mid \mathfrak{Q} X(z)=0, \mathfrak{Q} \in \mathcal{M}\}, & \Omega_{03}:=\left\{z \in \Omega \backslash \Omega_{01} \mid \operatorname{rank} A_{1}=1\right\}, \\
\Omega_{02}:=\{z \in \Omega \mid \mathfrak{Q} Y(z)=0, \mathfrak{Q} \in \mathcal{M}\}, & \Omega_{04}:=\left\{z \in \Omega \backslash \Omega_{02} \mid \operatorname{rank} A_{2}=1\right\} .
\end{array}
$$

If the set $\Omega_{01}$ (resp. $\Omega_{02}$ ) contains an open subset $\mathcal{U}$, then the row of $\mathrm{J}$ that is associated with $X$ (resp. $Y$ ) and thus the Jacobian J itself would vanish on $\mathcal{U}$, which is a contradiction. Therefore, the subsets $\Omega_{01}$ and $\Omega_{02}$ are nowhere dense. Suppose that the set $\Omega_{03}$ contains an open subset $\mathcal{U}$. Then there exists $\Lambda \in \mathbb{C}^{\infty}(\mathcal{U}, \mathbb{R})$ such that $\mathfrak{Q} U^{x}-\left(\Phi^{*} \tilde{f}\right) \mathfrak{Q} Y=\Lambda \mathfrak{Q} X$ on $\mathcal{U}$ for any $\mathfrak{Q} \in \mathcal{M}$, which implies the equations $\mathrm{D}_{x} U^{x}=\Lambda \mathrm{D}_{x} X+\left(\Phi^{*} \tilde{f}\right) \mathrm{D}_{x} Y$ and $\mathrm{D}_{y} U^{x}=\Lambda \mathrm{D}_{y} X+\left(\Phi^{*} \tilde{f}\right) \mathrm{D}_{y} Y$ on $\mathcal{U}$. We respectively subtract these equations from the equalities $\mathrm{D}_{x} U^{x}=U^{x x} \mathrm{D}_{x} X+U^{x y} \mathrm{D}_{x} Y$ and $\mathrm{D}_{y} U^{x}=U^{x x} \mathrm{D}_{y} X+U^{x y} \mathrm{D}_{y} Y$, where $U^{x x}:=\Phi_{(2)}{ }^{*} \tilde{u}_{\tilde{x} \tilde{x}}, U^{x y}:=\Phi_{(2)}{ }^{*} \tilde{u}_{\tilde{x} \tilde{y}}$, and $\Phi_{(2)}$ denotes the prolongation of the contact transformation $\Phi$ to the jet space $\mathrm{J}^{2}\left(\mathbb{R}_{x, y}^{2}, \mathbb{R}_{u}\right)$. As a result, we derive the equations

$$
\left(U^{x x}-\Lambda\right) \mathrm{D}_{x} X+\left(U^{x y}-\Phi^{*} \tilde{f}\right) \mathrm{D}_{x} Y=0, \quad\left(U^{x x}-\Lambda\right) \mathrm{D}_{y} X+\left(U^{x y}-\Phi^{*} \tilde{f}\right) \mathrm{D}_{y} Y=0
$$

on $\mathcal{U}$. Restricting the equation (6) on the manifold defined by $\mathcal{E}_{f}$ in $\mathrm{J}^{2}\left(\mathbb{R}_{x, y}^{2}, \mathbb{R}_{u}\right)$, where $u_{x y}=f$ and $U^{x y}=\Phi^{*} \tilde{f}$, leads to the equalities

$$
\left(U^{x x}-\Lambda\right)\left(\hat{\mathrm{D}}_{x} X+f X_{u_{y}}+X_{u_{x}} u_{x x}\right)=0, \quad\left(U^{x x}-\Lambda\right)\left(\hat{\mathrm{D}}_{y} X+f X_{u_{x}}+X_{u_{y}} u_{y y}\right)=0 .
$$

Since the derivative $\tilde{u}_{\tilde{x} \tilde{x}}$ is not constrained on the solution set of $\mathcal{E}_{\tilde{f}}$, this implies the equations $\hat{\mathrm{D}}_{x} X+f X_{u_{y}}+X_{u_{x}} u_{x x}=0$ and $\hat{\mathrm{D}}_{y} X+f X_{u_{x}}+X_{u_{y}} u_{y y}=0$, which can be split with respect to $u_{x x}$ and $u_{y y}$ into the condition $\mathfrak{Q} X(z)=0, \mathfrak{Q} \in \mathcal{M}$, on $\mathcal{U}$, contradicting the definition of $\Omega_{03}$. Therefore, the subset $\Omega_{03}$ (and, similarly, $\Omega_{04}$ ) is nowhere dense.

The splitting of the equation (5) with respect to $u_{x x}$ and $u_{y y}$ leads to the condition that the $2 \times 2$ minors of $A_{1}$ and $A_{2}$ associated with the pairs $\left(\mathfrak{Q}_{1}, \mathfrak{Q}_{2}\right)$ from the set $\mathcal{M}_{1} \times \mathcal{M}_{2}$, where

$$
\mathcal{M}_{1}:=\left\{\hat{\mathrm{D}}_{x}+f \partial_{u_{y}}, \partial_{u_{x}}\right\}, \quad \mathcal{M}_{2}:=\left\{\hat{\mathrm{D}}_{y}+f \partial_{u_{x}}, \partial_{u_{y}}\right\},
$$

vanish. This condition implies, in view of the condition $\operatorname{rank} A_{1}(z)=\operatorname{rank} A_{2}(z)=2$ on $\Omega_{1}$, the system

$$
\begin{array}{lll}
\left(\mathfrak{Q}_{1} X\right)\left(\mathfrak{Q}_{2} X\right)=0, & \left(\mathfrak{Q}_{1} X\right)\left(\mathfrak{Q}_{2} U^{x}-\left(\Phi^{*} \tilde{f}\right) \mathfrak{Q}_{2} Y\right)=0, & \left(\mathfrak{Q}_{1} U^{x}-\left(\Phi^{*} \tilde{f}\right) \mathfrak{Q}_{1} Y\right)\left(\mathfrak{Q}_{2} X\right)=0, \\
\left(\mathfrak{Q}_{1} Y\right)\left(\mathfrak{Q}_{2} Y\right)=0, & \left(\mathfrak{Q}_{1} Y\right)\left(\mathfrak{Q}_{2} U^{y}-\left(\Phi^{*} \tilde{f}\right) \mathfrak{Q}_{2} X\right)=0, & \left(\mathfrak{Q}_{1} U^{y}-\left(\Phi^{*} \tilde{f}\right) \mathfrak{Q}_{1} X\right)\left(\mathfrak{Q}_{2} Y\right)=0
\end{array}
$$

on $\Omega_{1}$ and thus, by continuity, on $\Omega$. Here the operator $\mathfrak{Q}_{i}$ runs through the set $\mathcal{M}_{i}, i=1,2$. Therefore, in each point of $\Omega_{1}$, we have either $\left(\mathfrak{Q}_{1} X\right)_{\mathfrak{Q}_{1} \in \mathcal{M}_{1}} \neq(0,0)$ or $\left(\mathfrak{Q}_{2} X\right)_{\mathfrak{Q}_{2} \in \mathcal{M}_{2}} \neq(0,0)$ and either $\left(\mathfrak{Q}_{1} Y\right)_{\mathfrak{Q}_{1} \in \mathcal{M}_{1}} \neq(0,0)$ or $\left(\mathfrak{Q}_{2} Y\right)_{\mathfrak{Q}_{2} \in \mathcal{M}_{2}} \neq(0,0)$.

If $\left(\mathfrak{Q}_{1} X\right)_{\mathfrak{Q}_{1} \in \mathcal{M}_{1}} \neq(0,0)$ at a point $z_{0} \in \Omega_{1}$, then $\left(\mathfrak{Q}_{2} Y\right)_{\mathfrak{Q}_{2} \in \mathcal{M}_{2}} \neq(0,0)$ at $z_{0}$. Indeed, otherwise $\left(\mathfrak{Q}_{1} X\right)_{\mathfrak{Q}_{1} \in \mathcal{M}_{1}} \neq(0,0)$ and $\left(\mathfrak{Q}_{1} Y\right)_{\mathfrak{Q}_{1} \in \mathcal{M}_{1}} \neq(0,0)$ simultaneously at $z_{0}$ and thus on 
some neighborhood $\mathcal{U}$ of $z_{0}$ in $\Omega_{1}$. This implies the equations $\mathfrak{Q}_{2} X=\mathfrak{Q}_{2} Y=0$ and then $\mathfrak{Q}_{2} U^{x}=\mathfrak{Q}_{2} U^{y}=0$ with $\mathfrak{Q}_{2} \in \mathcal{M}_{2}$. In particular, $X_{u_{y}}=Y_{u_{y}}=U_{u_{y}}^{x}=U_{u_{y}}^{y}=0$ and, in view of the third equation in (3), $U_{u_{y}}=0$, which contradicts the nondegeneracy of $\Phi$. It is obvious that the inverse implication, $\left(\mathfrak{Q}_{1} X\right)_{\mathfrak{Q}_{1} \in \mathcal{M}_{1}} \neq(0,0)$ at a point of $\Omega_{1}$ if $\left(\mathfrak{Q}_{2} Y\right)_{\mathfrak{Q}_{2} \in \mathcal{M}_{2}} \neq(0,0)$ at the same point, also holds true. Analogously, we also have that $\left(\mathfrak{Q}_{2} X\right)_{\mathfrak{Q}_{2} \in \mathcal{M}_{2}} \neq(0,0)$ at a point $z_{0} \in \Omega_{1}$ if and only if $\left(\mathfrak{Q}_{1} Y\right)_{\mathfrak{Q}_{1} \in \mathcal{M}_{1}} \neq(0,0)$ at $z_{0}$. Denoting

$$
\begin{aligned}
& \Xi_{1}:=\left\{z \in \Omega_{1} \mid\left(\mathfrak{Q}_{1} X\right)_{\mathfrak{Q}_{1} \in \mathcal{M}_{1}} \neq(0,0),\left(\mathfrak{Q}_{2} Y\right)_{\mathfrak{Q}_{2} \in \mathcal{M}_{2}} \neq(0,0)\right\}, \\
& \Xi_{2}:=\left\{z \in \Omega_{1} \mid\left(\mathfrak{Q}_{2} X\right)_{\mathfrak{Q}_{2} \in \mathcal{M}_{2}} \neq(0,0),\left(\mathfrak{Q}_{1} Y\right)_{\mathfrak{Q}_{1} \in \mathcal{M}_{1}} \neq(0,0)\right\},
\end{aligned}
$$

we get $\Omega_{1}=\Xi_{1} \sqcup \Xi_{2}$. On the closure $\operatorname{cl}\left(\Xi_{1}\right)$ of $\Xi_{1}$ in $\Omega$, the components of $\Phi$ satisfy the system (4). Note that on the subset, where $X_{u_{x}} \neq 0$ or $Y_{u_{y}} \neq 0$, the last equations in (4a) and in (4b) are differential consequences of the other equations in (4) supplemented with the equations (3). On the closure $\operatorname{cl}\left(\Xi_{2}\right)$ of $\Xi_{2}$ in $\Omega$, the components of $\Phi$ satisfy the analogous system obtained from the system (4) by permutation of $\left(x, u_{x}\right)$ and $\left(y, u_{y}\right)$. The sets $\operatorname{cl}\left(\Xi_{1}\right)$ and $\operatorname{cl}\left(\Xi_{2}\right)$ are disjoint since otherwise $\mathrm{J}=0$ on $\operatorname{cl}\left(\Xi_{1}\right) \cap \operatorname{cl}\left(\Xi_{2}\right)$. Therefore, $\Omega=\operatorname{cl}\left(\Omega_{1}\right)=\operatorname{cl}\left(\Xi_{1} \sqcup \Xi_{2}\right)=\operatorname{cl}\left(\Xi_{1}\right) \sqcup \operatorname{cl}\left(\Xi_{2}\right)$, which implies that both the sets $\operatorname{cl}\left(\Xi_{1}\right)$ and $\operatorname{cl}\left(\Xi_{2}\right)$ are open and closed in $\Omega$, and hence one of the sets is empty and the other coincides with $\Omega$. Up to the discrete equivalence transformation $\mathrm{J}^{0}$, we can assume that $\operatorname{cl}\left(\Xi_{1}\right)=\Omega$ and $\operatorname{cl}\left(\Xi_{2}\right)=\varnothing$, i.e., the system (4) holds on the entire set $\Omega$.

\section{Description of equivalence groupoids}

We first compute the point equivalence groupoid of the class $\mathcal{H}_{\text {gen }}$ and then single out its three disjoint subclasses, $\mathcal{H}_{x y}:=\mathcal{H}_{x} \cap \mathcal{H}_{y}, \mathcal{H}_{x}^{\prime}:=\mathcal{H}_{x} \backslash \mathcal{H}_{y}$ and $\mathcal{H}_{y}^{\prime}:=\mathcal{H}_{y} \backslash \mathcal{H}_{x}$, where

$$
\begin{aligned}
& \mathcal{H}_{x}:=\left\{\mathcal{E}_{f} \in \mathcal{H}_{\text {gen }} \mid f=F^{0}\left(x, y, u, u_{y}\right)+F^{1}\left(x, y, u, u_{y}\right) u_{x}, F_{x}^{1}+F^{0} F_{u_{y}}^{1}=F_{u}^{0}+F^{1} F_{u_{y}}^{0}\right\}, \\
& \mathcal{H}_{y}:=\left\{\mathcal{E}_{f} \in \mathcal{H}_{\text {gen }} \mid f=F^{0}\left(x, y, u, u_{x}\right)+F^{1}\left(x, y, u, u_{x}\right) u_{y}, F_{y}^{1}+F^{0} F_{u_{x}}^{1}=F_{u}^{0}+F^{1} F_{u_{x}}^{0}\right\},
\end{aligned}
$$

and hence

$$
\begin{aligned}
\mathcal{H}_{x y}=\left\{\mathcal{E}_{f} \in \mathcal{H}_{\text {gen }} \mid f=f^{0}(x, y, u)+f^{1}(x, y, u) u_{x}+f^{2}(x, y, u) u_{y}+f^{3}(x, y, u) u_{x} u_{y},\right. \\
\left.f_{y}^{3}=f_{u}^{1}, f_{x}^{3}=f_{u}^{2}, f_{y}^{2}=f_{x}^{1}=f_{u}^{0}+f^{1} f^{2}-f^{3} f^{0}\right\} .
\end{aligned}
$$

We will prove that any contact admissible transformation in $\mathcal{H}_{\text {gen }}$ that is not the first-order prolongation of a point admissible transformation in $\mathcal{H}_{\text {gen }}$ belongs to the contact equivalence groupoid of $\mathcal{H}_{x} \cup \mathcal{H}_{y}$. It is obvious that the subclasses $\mathcal{H}_{x}$ and $\mathcal{H}_{y}$ (resp. the subclasses $\mathcal{H}_{x}^{\prime}$ and $\mathcal{H}_{y}^{\prime}$ ) are mapped onto each other by the permutation $\mathcal{J}^{0}$. This is why any assertion that is true for one of these two subclasses holds true for the other after the modification using $\mathrm{J}^{0}$, and each of them can be substituted by the other in the assertions below. Any equation $\mathcal{E}_{f}$ from the subclass $\mathcal{H}_{x}$ (resp. $\mathcal{H}_{y}$ or $\mathcal{H}_{x y}$ ) can be (locally) represented in the form $\mathrm{D}_{x} g=0$ (resp. $\mathrm{D}_{y} h=0$ or $\left.\mathrm{D}_{x} \mathrm{D}_{y} \theta=0\right)$. Here $g=g\left(x, y, u, u_{y}\right), h=h\left(x, y, u, u_{x}\right)$ and $\theta=\theta(x, y, u)$ are arbitrary smooth functions of their arguments with $g_{u_{y}} \neq 0, h_{u_{x}} \neq 0$ and $\theta_{u} \neq 0$. (The representation for equations from the subclass $\mathcal{H}_{x y}$ follows from those for the subclasses $\mathcal{H}_{x}$ and $\mathcal{H}_{y}$ in view of the theorem on null divergences [8, Theorem 4.24].) This is why all the equations from the subclasses $\mathcal{H}_{x}$ and $\mathcal{H}_{y}$, not to mention their intersection $\mathcal{H}_{x y}$, are Darboux integrable in a trivial way. The reparameterized class $\mathcal{H}_{y}$, where $h$ is assumed as the arbitrary element instead of $f$, possesses gauge equivalence transformations (see the definition of this notion in [11])

$$
\left(\tilde{x}, \tilde{y}, \tilde{u}, \tilde{u}_{\tilde{x}}, \tilde{u}_{\tilde{y}}\right)=\left(x, y, u, u_{x}, u_{y}\right), \quad \tilde{h}=H(x, h),
$$

where $H$ is an arbitrary smooth function of $(x, h)$ with $H_{h} \neq 0$. 
Theorem 2. (i) The class $\mathcal{H}_{\text {gen }}$ is normalized in the usual sense with respect to point transformations. Its point equivalence group $G_{\text {gen }}^{\sim}$ coincides with its contact equivalence group and is generated by the discrete equivalence transformation $\mathrm{J}^{0}: \tilde{x}=y, \tilde{y}=x, \tilde{u}=u, \tilde{u}_{\tilde{x}}=u_{y}, \tilde{u}_{\tilde{y}}=u_{x}$, $\tilde{f}=f$ and the transformations of the form

$$
\begin{aligned}
& \tilde{x}=X(x), \quad \tilde{y}=Y(y), \quad \tilde{u}=U(x, y, u), \quad \tilde{u}_{\tilde{x}}=\frac{U_{x}+U_{u} u_{x}}{X_{x}}, \quad \tilde{u}_{\tilde{y}}=\frac{U_{y}+U_{u} u_{y}}{Y_{y}}, \\
& \tilde{f}=\frac{1}{X_{x} Y_{y}}\left(U_{u} f+U_{x y}+U_{x u} u_{y}+U_{y u} u_{x}+U_{u u} u_{x} u_{y}\right),
\end{aligned}
$$

where $X, Y$ and $U$ are arbitrary smooth functions of their arguments with $X_{x} Y_{y} U_{u} \neq 0$.

(ii) The contact equivalence groupoid $\mathcal{G}_{\text {gen }}^{\sim \mathrm{c}}$ of the class $\mathcal{H}_{\text {gen }}$ is generated by

- the first-order prolongation of the point equivalence groupoid $\mathcal{G}_{\text {gen }}^{\sim \mathrm{p}}$ of this class,

- the contact vertex group $\mathcal{G}_{0}^{\mathrm{c}}$ of the wave equation $\mathcal{E}_{0}: u_{x y}=0$ and

- the subgroupoid $\mathcal{G}_{y}^{\sim \mathrm{q}}$ of the equivalence groupoid $\mathcal{G}_{y}^{\sim \mathrm{c}}$ of the subclass $\mathcal{H}_{y}^{\prime}$ whose elements are induced, modulo gauge equivalence within the reparameterized class $\mathcal{H}_{y}^{\prime}$, by the pullbacks of the transformations of the form $\tilde{\tau}=\eta, \tilde{\xi}=\xi, \tilde{v}=\Upsilon(\tau, \xi, v, \eta), \tilde{\eta}=\tau$ with $\Upsilon_{v} \neq 0$ in the space with the coordinates $(\tau, \xi, v, \eta)$ by the mapping $\Psi:(\tau, \xi, v)=(x, y, u), \eta=$ $h\left(x, y, u, u_{x}\right)$, where $\tilde{u}_{\tilde{x}}=\Psi^{*} \Upsilon_{\eta}, \tilde{u}_{\tilde{y}}=\Psi^{*} \Upsilon_{\xi}+u_{y} \Psi^{*} \Upsilon_{v}$, and $h$ is an arbitrary element of the reparameterized class $\mathcal{H}_{y}^{\prime}$.

The subclasses $\mathcal{H}_{x y}, \mathcal{C}_{0}:=\mathcal{H}_{x} \triangle \mathcal{H}_{y}=\mathcal{H}_{x}^{\prime} \cup \mathcal{H}_{y}^{\prime}$ and $\mathcal{C}_{1}:=\mathcal{H}_{\text {gen }} \backslash\left(\mathcal{H}_{x} \cup \mathcal{H}_{y}\right)$ are invariant under the action of $\mathcal{G}_{\text {gen }}^{\sim \mathrm{c}}$. In other words, the partition of the class $\mathcal{H}_{\text {gen }}$ into these three subclasses, $\mathcal{H}_{\text {gen }}=\mathcal{H}_{x y} \sqcup \mathcal{C}_{0} \sqcup \mathcal{C}_{1}$, induces the corresponding partitions of its contact equivalence groupoid, $\mathcal{G}_{\text {gen }}^{\sim \mathrm{c}}=\mathcal{G}_{\mathcal{H}_{x y}}^{\sim \mathrm{c}} \sqcup \mathcal{G}_{\mathcal{C}_{0}}^{\sim \mathrm{c}} \sqcup \mathcal{G}_{\mathcal{C}_{1}}^{\sim \mathrm{c}}$.

(iii) The $\mathcal{G}_{\text {gen }}^{\sim \mathrm{c}}$-orbit of the wave equation $\mathcal{E}_{0}$ coincides with the subclass $\mathcal{H}_{x y}$.

(iv) The contact symmetry group $G_{0}^{\mathrm{c}}$ of $\mathcal{E}_{0}$ is generated by the discrete permutation transformation $\tilde{x}=y, \tilde{y}=x, \tilde{u}=u, \tilde{u}_{\tilde{x}}=u_{y}, \tilde{u}_{\tilde{y}}=u_{x}$ and the contact transformations of the form

$$
\begin{aligned}
& \tilde{x}=X\left(x, u_{x}\right), \quad \tilde{y}=Y\left(y, u_{y}\right), \quad \tilde{u}=c u+U^{1}\left(x, u_{x}\right)+U^{2}\left(y, u_{y}\right), \\
& \tilde{u}_{\tilde{x}}=U^{x}\left(x, u_{x}\right), \quad \tilde{u}_{\tilde{y}}=U^{y}\left(y, u_{y}\right),
\end{aligned}
$$

where $X, Y, U^{1}$ and $U^{2}$ are arbitrary smooth functions of their arguments and $c$ is an arbitrary constant with $\left(X_{x}, X_{u_{x}}\right) \neq(0,0),\left(Y_{y}, Y_{u_{y}}\right) \neq(0,0), c \neq 0$ and

$$
U_{u_{x}}^{1}=U^{x} X_{u_{x}}, \quad c u_{x}+U_{x}^{1}=U^{x} X_{x}, \quad U_{u_{y}}^{2}=U^{y} Y_{u_{y}}, \quad c u_{y}+U_{y}^{2}=U^{y} Y_{y} .
$$

Proof. We sketch the proof using the statement, the notation and the proof of Lemma 1 and the equivalence generated by the transformation $\mathrm{J}^{0}$. Looking for contact equivalence transformations of the class $\mathcal{H}_{\text {gen }}$, we split, with respect to the source and target values of the arbitrary element, $f$ and $\tilde{f}$, the equations of the system (4) that do not involve these values simultaneously. Taking into account the contact condition (3), we obtain the system

$$
\begin{aligned}
& X_{u_{x}}=X_{u_{y}}=X_{u}=X_{y}=0, \quad Y_{u_{x}}=Y_{u_{y}}=Y_{u}=Y_{x}=0, \quad U_{u_{x}}=U_{u_{y}}=0, \\
& U^{x}=\frac{\hat{\mathrm{D}}_{x} U}{X_{x}}, \quad U^{y}=\frac{\hat{\mathrm{D}}_{y} U}{Y_{y}},
\end{aligned}
$$

where $X_{x} Y_{y} U_{u} \neq 0$ since $\mathrm{J} \neq 0$. In addition, we derive the equation (7b) defining the $f$-components of equivalence transformations. Any point admissible transformation in the class $\mathcal{H}_{\text {gen }}$ relates its source and target according to $(7 \mathrm{~b})$, its transformational part satisfies the equations (10a) and thus it is induced by a point equivalence transformation of the class $\mathcal{H}_{\text {gen }}$. This proves point (i) of the theorem. 
Under the constraint $X_{u_{x}}=Y_{u_{y}}=0$, the systems (3) and (4) imply the system (10), i.e., any contact admissible transformation whose $x$ - and $y$-components do not depend respectively on $u_{x}$ and on $u_{y}$ is the first-order prolongation of a point admissible transformation.

If both $f=0$ and $\tilde{f}=0$, then the joint system (3)-(4) reduces to $X_{u}=X_{y}=0, Y_{u}=Y_{x}=0$, $U_{u_{y}}^{x}=U_{u}^{x}=U_{y}^{x}=0, U_{u_{x}}^{y}=U_{u}^{y}=U_{x}^{y}=0, U^{x} X_{u_{x}}=U_{u_{x}}, U^{x} \hat{\mathrm{D}}_{x} X=\hat{\mathrm{D}}_{x} U, U^{y} Y_{u_{y}}=U_{u_{y}}$, $U^{y} \hat{\mathrm{D}}_{y} Y=\hat{\mathrm{D}}_{y} U$, where $\left(X_{x}, X_{u_{x}}\right) \neq(0,0),\left(Y_{y}, Y_{u_{y}}\right) \neq(0,0)$ and $U_{u} \neq 0$ in view of $\mathrm{J} \neq 0$, which implies $U_{u_{x} u_{y}}=U_{u_{x} u}=U_{u_{x} y}=U_{u_{y} u}=U_{u_{y} x}=0$, and hence point (iv) holds true.

If just $f=0$, then similarly we derive $X_{u}=X_{y}=0$ and $Y_{u}=Y_{x}=0$, i.e., $\tilde{x}=X\left(x, u_{x}\right)$ and $\tilde{y}=Y\left(y, u_{y}\right)$ with $\left(X_{x}, X_{u_{x}}\right) \neq(0,0)$ and $\left(Y_{y}, Y_{u_{y}}\right) \neq(0,0)$. Factoring out the element of $\mathcal{G}_{0}^{\mathrm{c}}$ with the same $x$ - and $y$-components as $\mathcal{T}$ and with the identity $u$-component, $\tilde{u}=u$, from $\mathcal{T}$, we can set $X=x$ and $Y=y$, and then $U=U(x, y, u)$. This makes point (iii) obvious. Moreover, it becomes clear that the subclass $\mathcal{H}_{x y}$ is invariant under the action of $\mathcal{G}_{\text {gen }}^{\sim c}$.

If both $X_{u_{x}} \neq 0$ and $Y_{u_{y}} \neq 0$, then the first equations in (4a) and (4b) immediately imply $f_{u_{x} u_{x}}=f_{u_{y} u_{y}}=0$ and, after splitting them with respect to $u_{x}$ and $u_{y}$ and testing the obtained equations on the compatibility with respect to $X$ and $Y$, give the other constraints on $f$, jointly meaning that $\mathcal{E}_{f} \in \mathcal{H}_{x y}$. In other words, the $(x, y)$-components of $\mathcal{T} \in \mathcal{G}_{\text {gen }}^{\sim \mathrm{c}}$ simultaneously depend on the first derivatives of $u$ only if $\mathcal{T} \in \mathcal{G}_{\mathcal{H}}^{\sim} \mathrm{c}$.

Suppose that only one of the derivatives $X_{u_{x}}$ and $Y_{u_{y}}$ does not vanish. Up to the equivalence generated by $\mathrm{J}^{0}$, we can assume that $X_{u_{x}} \neq 0$ and $Y_{u_{y}}=0$. Then we derive from the joint system (3)-(4) that

$$
\begin{aligned}
& X=X\left(x, y, u, u_{x}\right), \quad Y=Y(y), \quad U=U\left(x, y, u, u_{x}\right), \quad \text { where } \quad U_{u_{x}} \hat{\mathrm{D}}_{x} X=X_{u_{x}} \hat{\mathrm{D}}_{x} U, \\
& U^{x}=\frac{U_{u_{x}}}{X_{u_{x}}}, \quad U^{y}=\frac{\hat{\mathrm{D}}_{y} U-U^{x} \hat{\mathrm{D}}_{y} X}{Y_{y}}, \quad \tilde{f}=\frac{U_{u_{x}}^{y}}{X_{u_{x}}}, \quad f=\frac{\hat{\mathrm{D}}_{y} X}{X_{u_{x}}} .
\end{aligned}
$$

The components of the inverse $\mathcal{T}^{-1}$ of $\mathcal{T}$ are of the same form as the respective components of $\mathcal{T}$. In particular, the derivative of the $x$-component of $\mathcal{T}^{-1}$ with respect to $u_{x}$ does not vanish as well since otherwise the admissible transformation $\mathcal{T}^{-1}$ and thus the admissible transformation $\mathcal{T}$ are the first-order prolongations of point admissible transformations in the class $\mathcal{H}_{\text {gen }}$, which contradicts the condition $X_{u_{x}} \neq 0$. This is why the first equation in (4a) implies that both the equations $\mathcal{E}_{f}$ and $\mathcal{E}_{\tilde{f}}$ belong to the class $\mathcal{H}_{y}$. Together with point (i) and the proven $\mathcal{G}_{\text {gen }^{-}}^{\sim c}$ invariance of $\mathcal{H}_{x y}$, this implies the $\mathcal{G}_{\text {gen }}^{\sim \mathrm{c}}$-invariance of $\mathcal{H}_{x} \cup \mathcal{H}_{y}$ and, hence, of $\mathcal{C}_{0}$ and of $\mathcal{C}_{1}$. We can set $Y=y$ up to the s- $G_{\text {gen }}^{\sim}$-equivalence on $\mathcal{G}_{\text {gen }}^{\sim \mathrm{c}}$. Then, taking $X$ as a value of the arbitrary element $h$ for the reparameterized form $\mathrm{D}_{y} h=0$ of the equation $\mathcal{E}_{f}$, we obtain that $\mathcal{T} \in \mathcal{G}_{y}^{\sim \mathrm{q}}$.

Remark 3. We explain, reformulate and expand the points of Theorem 2.

(i) The normalization of the class $\mathcal{H}_{\text {gen }}$ in the usual sense with respect to point transformations means that its point equivalence groupoid $\mathcal{G}_{\text {gen }}^{\sim \text { p }}$ coincides with the action groupoid $\mathcal{G}^{G \text { gen }}$ of the group $G_{\text {gen }}^{\sim}$, i.e., any point admissible transformation within the class $\mathcal{H}_{\text {gen }}$ is induced by its point equivalence transformation; see definitions in $[10,11,13]$.

(ii) Two equations from the class $\mathcal{C}_{1}$ are related by a contact transformation if and only if this transformation is the first-order prolongation of a point transformation that is the projection of an element of the group $G_{\text {gen }}^{\sim}$ to the space with coordinates $(x, y, u)$. In other words, the class $\mathcal{C}_{1}$ is normalized in the usual sense with respect to point transformations, its point equivalence group coincides with $G_{\text {gen }}^{\sim}$ and with its contact equivalence group, and its contact equivalence groupoid is the first-order prolongation of its point equivalence groupoid.

(iii) Let $G_{\text {gen }}^{\sim u}$ denote the subgroup of $G_{\text {gen }}^{\sim}$ that is constituted by the transformations of the form $(7)$ with $X(x)=x$ and $Y(y)=y$. The $\mathcal{G}_{\text {gen }}^{\sim \text { corbit }} \mathcal{H}_{x y}$ of the equation $\mathcal{E}_{0}$ coincides with its $G_{\text {gen }}^{\sim}$-orbit and, more specifically, with its $G_{\text {gen }}^{\sim \text { u}}$-orbit.

An equation $\mathcal{E}_{f}$ from the class $\mathcal{H}_{\text {gen }}$ reduces to $\mathcal{E}_{0}$ by a contact transformation if and only if it reduces to $\mathcal{E}_{0}$ by an element of $G_{\text {gen }}^{\sim \text { u }}$. Then the corresponding value of the arbitrary element $f$ is necessarily of the form $f=\left(\theta_{x y}+\theta_{x u} u_{y}+\theta_{y u} u_{x}+\theta_{u u} u_{x} u_{y}\right) / \theta_{u}$, where $\theta$ is a smooth function of $(x, y, u)$ with $\theta_{u} \neq 0$. 
The contact equivalence groupoid of the class $\mathcal{H}_{x y}$ is generated by the contact vertex group $\mathcal{G}_{0}^{\mathrm{c}}$ of the wave equation $\mathcal{E}_{0}$ and the restriction of the action groupoid of the group $G_{\text {gen }}^{\sim u}$ to $\mathcal{H}_{x y}$.

(iv) In view of (9), the $u_{x}$-component (resp. the $u_{y}$-component) of a contact symmetry transformation of $\mathcal{E}_{0}$ can be locally expressed in terms of the $x$ - and the $u$-components (resp. the $y$ and the $u$-components). More specifically, $U^{x}=U_{u_{x}}^{1} / X_{u_{x}}, U^{x}=\left(c u_{x}+U_{x}^{1}\right) / X_{x}, U^{y}=U_{u_{y}}^{2} / Y_{u_{y}}$ and $U^{y}=\left(c u_{y}+U_{y}^{2}\right) / Y_{y}$, whenever $X_{u_{x}} \neq 0, X_{x} \neq 0, Y_{u_{y}} \neq 0$ and $Y_{y} \neq 0$, respectively.

Excluding $U^{x}$ and $U^{y}$ from (9), we derive $U_{u_{x}}^{1} X_{x}=\left(c u_{x}+U_{x}^{1}\right) X_{u_{x}}$ and $U_{u_{y}}^{2} Y_{y}=\left(c u_{y}+U_{y}^{2}\right) Y_{u_{y}}$. Whenever $X_{u_{x}} \neq 0$, the former equation gives the following expression for $U^{1}$ :

$$
U^{1}\left(x, u_{x}\right)=-c \int_{t_{0}}^{x} \Theta^{1}\left(x^{\prime}, X\left(x, u_{x}\right)\right) \mathrm{d} x^{\prime}+\varphi^{1}\left(X\left(x, u_{x}\right)\right),
$$

where $\Theta^{1}$ is the inverse of the function $X$ with respect to $u_{x}, \Theta^{1}\left(x, X\left(x, u_{x}\right)\right)=u_{x}$, and $\varphi^{1}$ is an arbitrary smooth function of its single argument. Similarly, the later equation integrates to an expression for $U^{2}$ whenever $Y_{u_{y}} \neq 0$,

$$
U^{2}\left(y, u_{y}\right)=-c \int_{y_{0}}^{y} \Theta^{2}\left(y^{\prime}, Y\left(y, u_{y}\right)\right) \mathrm{d} y^{\prime}+\varphi^{2}\left(Y\left(y, u_{y}\right)\right)
$$

where $\Theta^{2}$ is the inverse of the function $Y$ with respect to $u_{y}, \Theta^{2}\left(y, Y\left(y, u_{y}\right)\right)=u_{x}$, and $\varphi^{2}$ is an arbitrary smooth function of its single argument.

For contact symmetry transformation (8) of $\mathcal{E}_{0}$, the equation (9) implies that $U_{u_{r}}^{1}=0$ if $X_{u_{x}}=0$ and $U_{u_{y}}^{2}=0$ if $Y_{u_{y}}=0$. Therefore, such a transformation with $X_{u_{x}}=Y_{u_{y}}=0$ is the first-order prolongation of a point symmetry transformation of $\mathcal{E}_{0}$.

The point symmetry group $G_{0}$ of the wave equation $\mathcal{E}_{0}$ is generated by the discrete permutation transformation $\tilde{x}=y, \tilde{y}=x, \tilde{u}=u$ and the point transformations of the form $\tilde{x}=X(x)$, $\tilde{y}=Y(y), \tilde{u}=c u+U^{1}(x)+U^{2}(y)$, where $X, Y, U^{1}$ and $U^{2}$ are arbitrary smooth functions of their arguments with $X_{x} \neq 0$ and $Y_{y} \neq 0$, and $c$ is an arbitrary nonzero constant. Therefore, a complete list of discrete point symmetry transformations of the equation $\mathcal{E}_{0}$ that are independent up to combining with each other and with continuous point symmetry transformations of this equation is exhausted by the $(x, y)$-permutation and the three transformations each of which alternates the sign of one of the variables $x, y$ and $u$. The quotient group of the point symmetry group $G_{0}$ of $\mathcal{E}_{0}$ with respect to its identity component is isomorphic to the group $\mathbb{Z}_{2} \times \mathbb{Z}_{2} \times \mathbb{Z}_{2} \times \mathbb{Z}_{2}$

Acknowledgments. The author thanks Vyacheslav Boyko, Michael Kunzinger and Dmytro Popovych for helpful discussions. The research was supported by the Austrian Science Fund (FWF), projects P25064 and P28770.

\section{References}

[1] Boyko V.M., Lokaziuk O.V. and Popovych R.O., Realizations of Lie algebras on the line and the new group classification of (1+1)-dimensional generalized nonlinear Klein-Gordon equations, arXiv:2008.05460, 30 pp.

[2] Kurujyibwami C. and Popovych R.O., Equivalence groupoids and group classification of multidimensional nonlinear Schrödinger equations, J. Math. Anal. Appl. 491 (2020), 124271, arXiv:2003.02781.

[3] Kushner A.G., On contact equivalence of Monge-Ampère equations to linear equations with constant coefficients, Acta Appl. Math. 109 (2010), 197-210.

[4] Kuznetsova M.N., Pekcan A., Zhiber A.V., The Klein-Gordon equation and differential substitutions of the form $v=\phi\left(u, u_{x}, u_{y}\right)$, SIGMA 8 (2012), Paper 090, arXiv:1111.7255.

[5] Lahno V. and Zhdanov R., Group classification of nonlinear wave equations, J. Math. Phys. 46 (2005), 053301.

[6] Lahno V., Zhdanov R. and Magda O., Group classification and exact solutions of nonlinear wave equations, Acta Appl. Math. 91 (2006), 253-313, arXiv:nlin.SI/0405069. 
[7] Lie S., Diskussion der Differentialgleichung $d^{2} z / d x d y=F(z)$, Arch. fur Math. 6 (1881), 112-124. (Reprinted in: Lie S., Gesammelte Abhandlungen, Vol. 3, B.G. Teubner, Leipzig and H. Aschehoug \& Co, Kristiania, 469-478.)

[8] Olver P.J., Application of Lie groups to differential equations, Springer, New York, 1993.

[9] Olver P.J., Equivalence, invariants, and symmetry, Cambridge University Press, Cambridge, 1995.

[10] Opanasenko S., Bihlo A. and Popovych R.O., Equivalence groupoid and group classification of a class of variable-coefficient Burgers equations, J. Math. Anal. Appl. 490 (2020), 124215, arXiv:1910.13500.

[11] Popovych R.O., Kunzinger M. and Eshraghi H., Admissible transformations and normalized classes of nonlinear Schrödinger equations, Acta Appl. Math. 109 (2010), 315-359, arXiv:math-ph/0611061.

[12] Stormark O., Lie's structural approach to PDE systems, Cambridge University Press, Cambridge, 2000.

[13] Vaneeva O.O., Bihlo A. and Popovych R.O., Generalization of the algebraic method of group classification with application to nonlinear wave and elliptic equations, Commun. Nonlinear Sci. Numer. Simul. 91 (2020), 105419, arXiv:2002.08939. 